\title{
First Look at Two-Loop Five-Gluon Scattering in QCD
}

\author{
Simon Badger, ${ }^{1, *}$ Christian Brønnum-Hansen, ${ }^{2}$ Heribertus Bayu Hartanto, ${ }^{1}$ and Tiziano Peraro ${ }^{3}$ \\ ${ }^{1}$ Institute for Particle Physics Phenomenology, Department of Physics, Durham University, Durham DH1 3LE, United Kingdom \\ ${ }^{2}$ Higgs Centre for Theoretical Physics, School of Physics and Astronomy, The University of Edinburgh, \\ Edinburgh EH9 3JZ, Scotland, United Kingdom \\ ${ }^{3}$ PRISMA Cluster of Excellence, Johannes Gutenberg University, 55128 Mainz, Germany
}

(Received 15 December 2017; published 2 March 2018)

\begin{abstract}
We compute the leading-color contributions to five-gluon scattering at two loops in massless QCD. The integrands of all independent helicity amplitudes are evaluated using $d$-dimensional generalized unitarity cuts and finite field reconstruction techniques. Numerical evaluation of the integral basis is performed with sector decomposition methods to obtain the first benchmark results for all helicity configurations of a $2 \rightarrow 3$ scattering process in QCD.
\end{abstract}

DOI: 10.1103/PhysRevLett.120.092001

Introduction.-As data continue to pour in from the LHC experiments, the precision of many theoretical predictions for high-energy scattering processes is being challenged by experimental measurements. While there has been remarkable progress in standard model predictions for multiparticle final states at next-to-leading order and $2 \rightarrow 2$ scattering processes at next-to-next-to-leading order (NNLO), the computational complexity of $2 \rightarrow 3$ scattering processes at NNLO results in many important measurements being currently (or in the near future) limited by theoretical uncertainties. Pure gluon scattering at two loops in QCD is a key bottleneck in making such predictions, which have been known for $g g \rightarrow g g$ for more than $15 \mathrm{yr}[1,2]$. The one-loop five-gluon amplitudes have been known since 1993 [3] and were among the first results from the on-shell methods that led to the modern unitarity method [4,5].

In this Letter, we demonstrate how new evaluation techniques based on generalized unitarity [6,7] and integrand reduction [8-14] can offer a solution to the traditional bottlenecks in these computations and present the first results for a complete set of planar five-gluon helicity amplitudes in QCD. The results extend previous results obtained for "all-plus" helicity amplitudes [15-22]. These on-shell techniques have also been explored in the context of maximal unitarity [23,24] and numerical unitarity [25-27] approaches to QCD amplitudes. Work in this area has received considerable interest due to the phenomenological importance of precision predictions for $2 \rightarrow 3$ scattering. Efforts to complete the unknown two-loop

Published by the American Physical Society under the terms of the Creative Commons Attribution 4.0 International license. Further distribution of this work must maintain attribution to the author(s) and the published article's title, journal citation, and DOI. Funded by SCOAP . amplitudes for processes such as $p p \rightarrow 3$ jets, $p p \rightarrow H+$ 2 jets, or $p p \rightarrow \gamma \gamma+$ jet have been further motivated by the recent analytic computations of the planar master integrals (MIs) $[18,28]$ using new differential equation techniques $[29,30]$.

Our approach exploits a parametrization of the multiparticle kinematics with rational functions combined with a numerical evaluation over finite fields [31] to avoid the large intermediate algebraic expressions that traditionally appear. The rational parametrization of the external kinematics is provided by momentum twistor coordinates [32].

Integrand parametrization and reconstruction.-We define the unrenormalized leading-color (planar) five-gluon amplitudes using the simple trace basis:

$$
\begin{aligned}
\mathcal{A}^{(L)}(1,2,3,4,5)= & n^{L} g_{s}^{3} \sum_{\sigma \in S_{5} / Z_{5}} \operatorname{tr}\left(T^{a_{\sigma(1)}} \ldots T^{a_{\sigma(5)}}\right) \\
& \times A^{(L)}(\sigma(1), \sigma(2), \sigma(3), \sigma(4), \sigma(5)),
\end{aligned}
$$

where $T^{a}$ are the fundamental generators of $S U\left(N_{c}\right)$ and $S_{5} / Z_{5}$ are all noncyclic permutations of the external particles. The overall normalization is $n=m_{\epsilon} N_{c} \alpha_{s} /(4 \pi)$, where $\alpha_{s}=g_{s}^{2} /(4 \pi)$ is the strong coupling constant and $m_{\epsilon}=i(4 \pi)^{\epsilon} e^{-\epsilon \gamma_{E}}\left(\gamma_{E}\right.$ is the Euler-Mascheroni constant). The $L$-loop partial amplitude $A^{(L)}$ can be constructed from color-ordered Feynman diagrams. In this Letter, we will compute the pure gluonic contributions to these amplitudes at two loops including the dependence on the spin dimension $d_{s}$. Results in the 't Hooft-Veltman and fourdimensional-helicity (FDH) schemes can be obtained by setting $d_{s}=4-2 \epsilon$ and $d_{s}=4$, respectively [33].

The integrand of the ordered partial amplitudes can be parametrized in terms of irreducible numerators $\Delta$ : 


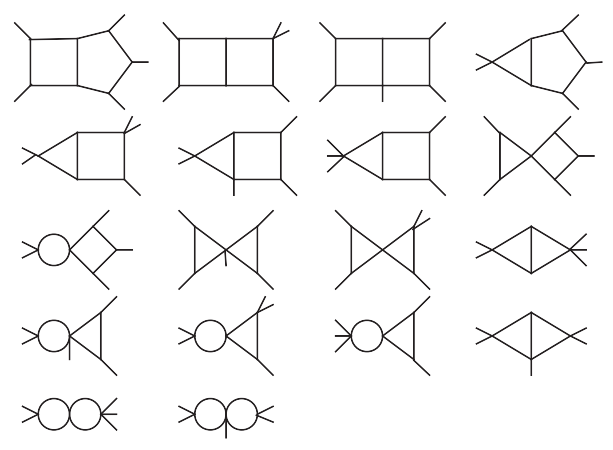

FIG. 1. The 18 distinct topologies extractable from (one-loop) ${ }^{2}$ cuts.

$A^{(2)}(1,2,3,4,5)=\int\left[d k_{1}\right]\left[d k_{2}\right] \sum_{T} \frac{\Delta_{T}(\{k\},\{p\})}{\prod_{\alpha \in T} D_{\alpha}}$,

where $\{k\}=\left\{k_{1}, k_{2}\right\}$ are the $(d=4-2 \epsilon)$-dimensional loop momenta, $T$ are the set of independent topologies, and $\{p\}=\{1,2,3,4,5\}$ are the ordered external momenta. The measure is $\left[d k_{i}\right]=-i \pi^{-d / 2} e^{\epsilon \gamma_{E}} d^{4-2 \epsilon} k_{i}$, and the index $\alpha$ runs over the set of propagators associated with the topology $T$. Our planar five-gluon amplitudes are built from 57 distinct topologies, giving 425 irreducible numerators when including permutations of the external legs. 18 of these 57 topologies can be extracted from the (one-loop) ${ }^{2}$ cut configurations as shown in Fig. 1. This means that all topologies with an additional propagator including $k_{1}+k_{2}$ are computed simultaneously with the (one-loop) ${ }^{2}$ cuts. This is more efficient, since the parametrizations of the cut loop momentum solutions are much simpler. The remaining 39 can be extracted from a further 31 configurations shown in Fig. 2. The eight topologies shown in Fig. 3 have divergent maximal cuts and are extracted simultaneously with subtopologies within the set of 31 two-loop cuts.

The construction of an integrand basis has been discussed before using the language of computational

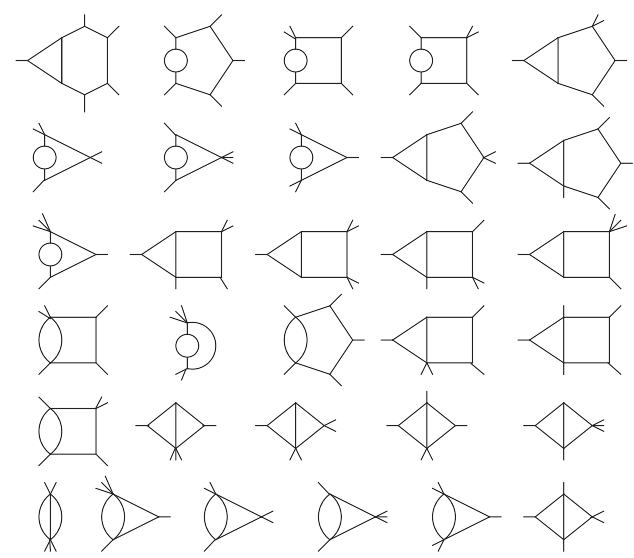

FIG. 2. The 31 distinct topologies extractable from two-loop cuts. algebraic geometry through polynomial division over a Gröbner basis $[10,14]$. In this work, we took a simpler approach, which did not rely on the computation of a Gröbner basis, instead relying on the inversion of a linear system which can be performed efficiently with finite field reconstruction methods. We begin by expanding the loop momenta around a basis of external momenta and transverse directions (similarly to the methods of van Neerven and Vermaseren [34]):

$$
k_{i}^{\mu}=k_{\|, i}^{\mu}+k_{\perp, i}^{\mu}
$$

where $k_{\|}$lives in the physical space spanned by the external momenta of the topology and $k_{\perp}$ lives in the transverse space. We further decompose the transverse space into four-dimensional and $(-2 \epsilon)$-dimensional spaces, $k_{\perp, i}=$ $k_{\perp, i}^{[4]}+k_{\perp, i}^{[-2 \epsilon]}$. The size of the 4D transverse space (which we will call the spurious space) has dimension $d_{\perp,[4]}=$ $4-d_{\|}$, where $d_{\|}$is equal to the number of independent momenta entering the vertices of the topology, up to a maximum value of four. We choose a spanning basis $v$ for the physical space of each topology $k_{\|, i}^{\mu}=\sum_{j=1}^{d_{\|}} a_{i j} v_{j}^{\mu}$ and a basis $w$ for the spurious space $k_{\perp, i}^{\mu,[4]}=\sum_{j=1}^{d_{\perp, 4]}} b_{i j} w_{j}^{\mu}$, with $v_{i} \cdot w_{j}=0$.

The coefficients in the physical space $k_{\|}$are functions of the $a_{i j}\left(k_{i}\right) \equiv a_{i j}(\{D\},\{k \cdot q\})$, where $D$ are the inverse propagators and $k_{i} \cdot q_{j}$ are the physical space irreducible scalar products (ISPs) for a given topology, where $q_{j}$ are suitable linear combinations of external momenta. The coefficients in the spurious and $(-2 \epsilon)$-dimensional spaces are functions of additional ISPs $k_{i} \cdot w_{j}$ and $\mu_{i j}=-k_{\perp, i}^{[-2 \epsilon]}$. $k_{\perp, j}^{[-2 \epsilon]}$. Having completed this decomposition, we find relations between monomials in the ISPs by expanding Eq. (3):

$$
\mu_{i j}=k_{i} \cdot k_{j}-k_{\|, i} \cdot k_{\|, j}-k_{\perp, i}^{[4]} \cdot k_{\perp, j}^{[4]} .
$$

From this equation, it is easy to obtain a valid basis of monomials for each irreducible numerator of a dimensionally regulated amplitude by using Eq. (4) to remove dependence on the extra dimensional ISPs. This basis is just the most general polynomial in the ISPs $k_{i} \cdot q_{j}$ and

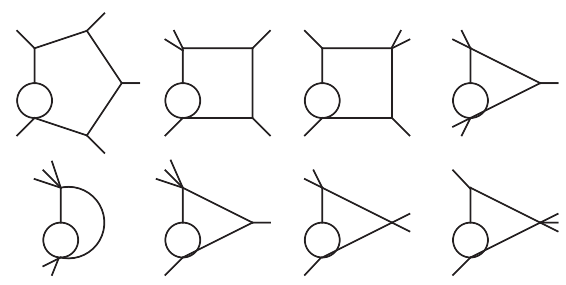

FIG. 3. The eight distinct topologies with divergent cuts that must be computed simultaneously with subtopologies. 
$k_{i} \cdot w_{j}$ where the power counting is restricted by the renormalizability constraints [35].

This simple basis, without dependence on $\mu_{i j}$ monomials, is trivial to obtain without polynomial division but results in high-rank tensor integrals with a complicated infrared (IR) pole structure. Instead, we prefer to map to a new basis which prefers to keep monomials in $\mu_{i j}$ in the numerator and make the $\epsilon \rightarrow 0$ limit easier to perform. The map to the new basis is performed in four steps: (i) Write down an overcomplete set of monomials in $k_{i} \cdot q_{j}, k_{i} \cdot w_{j}$, and $\mu_{i j}$ obeying the power counting restrictions; (ii) choose a set of criteria to order the overcomplete set of monomials (for example, prefer lower-rank monomials or prefer monomials proportional to $\mu_{i j}$ ); (iii) map each monomial containing $\mu_{i j}$ from the set of step (i) onto a linear combination of monomials of the simple basis using Eq. (4), to obtain a system of linear relations between monomials in the overcomplete set; (iv) solve for the independent monomials of this linear system to find the new basis. The result of this procedure is a processindependent basis of monomials whose coefficients can be fixed from unitarity cuts in six dimensions. We take a top-down, OPP-like approach (i.e. following the method of Ref. [8] by Ossola, Papadopoulos and Pittau) to solving the complete system using information from previously computed cuts to remove known poles from the factorized product of tree amplitudes using the six-dimensional spinor-helicity formalism [36]. The product of tree amplitudes is efficiently evaluated by sewing together BerendsGiele currents [37] as described in Ref. [31].

After completing the integrand level reconstruction, the remaining transverse integration must be performed to obtain a form compatible with traditional integration-byparts (IBP) relations. Following a recent approach [38], we have two options in order to achieve this: (i) to integrate the full transverse space to remove $k_{i} \cdot w_{j}$ and $\mu_{i j}$, introducing dependence in $\epsilon$ into the integral coefficients, or (ii) to integrate only over the spurious space, retaining $\mu_{i j}$ dependence which can subsequently be removed through dimension-shifting identities. In this work, we have taken the second approach, since it turned out to have better numerical stability to use dimension-shifted integrals instead of high-rank tensor integrals.

In either case, the tensor structure in the transverse space can involve only the metric tensor $g_{\perp}^{\mu \nu}$ (or $g_{\perp,[4]}^{\mu \nu}, g_{\perp_{:}[-2 \epsilon]}^{\mu \nu}$ depending on the particular transverse space being integrated out). This makes the tensor decomposition for nonvanishing integrals in the spurious space rather simple. Further examples of this technique can be found in Ref. [38].

As a final alteration of the integrand basis, we replace some monomials with spurious integrands by building in integration identities and symmetry relations (such as $k_{1} \leftrightarrow$ $k_{2}$ in the three-propagator sunrise topology). For example, rather than fitting the coefficient of $\left(k_{1} \cdot w_{2}\right)^{2}$, we replace it with the function

$$
\left(k_{1} \cdot w_{2}\right)^{2} \rightarrow\left(k_{1} \cdot w_{2}\right)^{2}-\frac{w_{2}^{2}}{d_{\perp,[4]}} k_{\perp, 1}^{[4]} \cdot k_{\perp, 1}^{[4]},
$$

which will integrate to zero. In Table I, we summarize the result of our fit to unitarity cuts listing the number of nonzero coefficients at the integrand level before and after performing the integration over the spurious space. Cuts with scalar loops are required for the reduction from six to $4-2 \epsilon$ dimensions. We perform the fit taking into account the individual contribution of these scalar loops in order to reconstruct the dependence of the numerator on the spin dimension $d_{s}$. Setting $d_{s}=2$ gives a supersymmetric limit in which the highest-rank tensor integrals do not appear in the amplitudes. We use a polynomial expansion of the integrand in $\left(d_{s}-2\right)$ to separate the coefficients into terms of increasing complexity. The fit can be performed efficiently using rational numerics for each phase-space point, and in most cases it was possible to obtain completely analytic expressions for the integrands of the helicity amplitudes using modest computing resources.

Numerical evaluation.-The unitarity-based method outlined above has been complemented by an approach based on a numerical evaluation of Feynman diagrams to determine the coefficients of independent monomial bases. Both of these methods use a momentum twistor [32] parametrization of the external kinematics to obtain a rational numerical phase-space point. This is extremely important, since in order to make use of the finite field reconstruction methods our numerical algorithm must be free of all square roots [39-42]. The parametrization in this case was chosen (somewhat arbitrarily) to be

$$
Z=\left(\begin{array}{ccccc}
1 & 0 & \frac{1}{x_{1}} & \frac{1+x_{2}}{x_{1} x_{2}} & \frac{1+x_{3}\left(1+x_{2}\right)}{x_{1} x_{2} x_{3}} \\
0 & 1 & 1 & 1 & 1 \\
0 & 0 & 0 & \frac{x_{4}}{x_{2}} & 1 \\
0 & 0 & 1 & 1 & \frac{x_{4}-x_{5}}{x_{4}}
\end{array}\right),
$$

where the columns give the four-component momentum twistors of the five external particles (see, for example, Appendix A of Ref. [15] for more details). These methods have been implemented using a combination of tools including QGRAF [43], FORM [44,45], Mathematica, and a private implementation of the finite field reconstruction method [31].

We have validated our setup on a number of known cases. First, we have reproduced integrand level expressions for the all-plus helicity sector [15] and against the known integrands in the $\mathcal{N}=4$ super-Yang-Mills theory [46]. The latter check was obtained by computing all fermion and (complex)-scalar loop contributions and subsequently setting $n_{f}=\mathcal{N}$ and $n_{s}=\mathcal{N}-1$. We also have performed gauge invariance checks at the integrand level using the Feynman diagram setup. 
TABLE I. The number of nonzero coefficients found at the integrand level both before ("nonzero") and after ("nonspurious") removing monomials which integrate to zero. The last column ["contributions @ $\mathcal{O}\left(\epsilon^{0}\right)$ "] gives the number of coefficients contributing to the finite part. Each helicity amplitude is split into the components of $d_{s}-2$.

\begin{tabular}{lccrr}
\hline \hline Helicity & Flavor & Nonzero coefficients & Nonspurious coefficients & Contributions @ O $\left(\epsilon^{0}\right)$ \\
\hline+++++ & $\left(d_{s}-2\right)^{0}$ & 50 & 50 & 0 \\
& $\left(d_{s}-2\right)^{1}$ & 175 & 165 & 50 \\
-++++ & $\left(d_{s}-2\right)^{2}$ & 320 & 90 & 60 \\
& $\left(d_{s}-2\right)^{0}$ & 1153 & 761 & 405 \\
-++++ & $\left(d_{s}-2\right)^{1}$ & 8745 & 4020 & 3436 \\
& $\left(d_{s}-2\right)^{2}$ & 1037 & 100 & 68 \\
& $\left(d_{s}-2\right)^{0}$ & 2234 & 1267 & 976 \\
-+++ & $\left(d_{s}-2\right)^{1}$ & 11844 & 5342 & 4659 \\
& $\left(d_{s}-2\right)^{2}$ & 1641 & 71 & 48 \\
& $\left(d_{s}-2\right)^{0}$ & 3137 & 1732 & 1335 \\
& $\left(d_{s}-2\right)^{1}$ & 15282 & 6654 & 5734 \\
\hline \hline
\end{tabular}

To obtain a numerical value for the complete amplitude after integration, we perform a sector decomposition of the basis integrals combined with a Monte Carlo integration. After applying dimension-shifting relations $[2,47,48]$ to rewrite the extra dimensional ISPs as standard integrals, we processed the full set of integrals using both FIESTA [49] and PYSECDEC [50] packages. This setup was validated with the four-gluon helicity amplitudes and cross-checked against results in the literature [25]. Simple topologies with $2 \rightarrow 2$ kinematics were reduced to the known MIs of Ref. [51] using IBPs from FIRE5 [52] and REDUZE2 [53] and dimensional recurrence relations from LITERED [54]. This gave a substantial improvement in the numerical accuracy.

The results for evaluation at a specific phase-space point are given in Tables II and III for the amplitudes

$$
\hat{A}_{\lambda_{1} \lambda_{2} \lambda_{3} \lambda_{4} \lambda_{5}}^{(2)[i]}=\frac{A^{(2),[i]}\left(1^{\lambda_{1}}, 2^{\lambda_{2}}, 3^{\lambda_{3}}, 4^{\lambda_{4}}, 5^{\lambda_{5}}\right)}{A^{\mathrm{LO}}\left(1^{\lambda_{1}}, 2^{\lambda_{2}}, 3^{\lambda_{3}}, 4^{\lambda_{4}}, 5^{\lambda_{5}}\right)}
$$

TABLE II. The numerical evaluation of $\hat{A}^{(2),[0]}(1,2,3,4,5)$ using $\left\{x_{1}=-1, x_{2}=79 / 90, x_{3}=16 / 61, x_{4}=37 / 78, x_{5}=83 / 102\right\}$ in Eq. (6). The comparison with the universal pole structure $P$ is shown. The +++++ and -++++ amplitudes vanish to $\mathcal{O}(\epsilon)$ for this $\left(d_{s}-2\right)^{0}$ component.

\begin{tabular}{lccccc}
\hline \hline & $\epsilon^{-4}$ & $\epsilon^{-3}$ & $\epsilon^{-2}$ & $\epsilon^{-1}$ & $\epsilon^{0}$ \\
\hline$\hat{A}_{--+++}^{(2),[0]}$ & 12.5 & 27.7526 & -23.773 & -168.117 & $-175.207 \pm 0.004$ \\
$P_{--+++}^{(2),[0]}$ & 12.5 & 27.7526 & -23.773 & -168.116 & $\ldots$ \\
$\hat{A}_{-,[+++}^{(2),[0]}$ & 12.5 & 27.7526 & 2.5029 & -35.8094 & $69.661 \pm 0.009$ \\
$P_{-+-++}^{(2),[0]}$ & 12.5 & 27.7526 & 2.5028 & -35.8086 & $\cdots$ \\
\hline \hline
\end{tabular}

with helicities $\lambda_{i}$ and $A^{(2)}=\sum_{i=0}^{2}\left(d_{s}-2\right)^{i} A^{(2),[i]}$. The leading-order amplitudes $A^{\mathrm{LO}}$ are the tree level for the - +++ and -+-++ and rational one-loop amplitudes for the +++++ and -++++ . The finite (one-loop) $)^{2}$ configuration $A^{(2),[2]}$ is presented in Table IV. Numerical accuracy is not an issue here, since the integrand level reduction already leads to a basis of one-loop MIs. In addition, we find complete agreement with the finite part of the known integrated all-plus amplitude [18].

In cases where the $\epsilon$ pole structure of the amplitudes is nontrivial, we compared with the known universal IR structure [55-58] including the dependence on $d_{s}$ extracted from the FDH scheme results [59]. The leading pole in $1 / \epsilon^{4}$ was verified analytically and is therefore quoted exactly in Tables II and III. By comparing the agreement in the poles between the $\left(d_{s}-2\right)^{0}$ and $\left(d_{s}-2\right)^{1}$, we clearly see the

TABLE III. The numerical evaluation of $\hat{A}^{(2),[1]}(1,2,3,4,5)$ and comparison with the universal pole structure $P$ at the same kinematic point of Table II.

\begin{tabular}{lccccc}
\hline \hline & $\epsilon^{-4}$ & $\epsilon^{-3}$ & $\epsilon^{-2}$ & $\epsilon^{-1}$ & $\epsilon^{0}$ \\
\hline$\hat{A}_{++++++}^{(2),[1]}$ & 0 & 0.0000 & -2.5000 & -6.4324 & $-5.311 \pm 0.000$ \\
$P_{++++++}^{(2),[1]}$ & 0 & 0 & -2.5000 & -6.4324 & $\ldots$ \\
$\hat{A}_{-+++++}^{(2),[1]}$ & 0 & 0.0000 & -2.5000 & -12.749 & $-22.098 \pm 0.030$ \\
$P_{-+++++}^{(2),[1]}$ & 0 & 0 & -2.5000 & -12.749 & $\ldots$ \\
$\hat{A}_{--++++}^{(2),[1]}$ & 0 & -0.6250 & -1.8175 & -0.4871 & $3.127 \pm 0.030$ \\
$P_{--++++}^{(2),[1]}$ & 0 & -0.6250 & -1.8175 & -0.4869 & $\ldots$ \\
$\hat{A}_{-+,+++}^{(2),[1]}$ & 0 & -0.6249 & -2.7761 & -5.0017 & $0.172 \pm 0.030$ \\
$P_{-+-+++}^{(2),[1]}$ & 0 & -0.6250 & -2.7759 & -5.0018 & $\ldots$ \\
\hline \hline
\end{tabular}


TABLE IV. The numerical evaluation of finite $\hat{A}^{(2),[2]}(1,2$, $3,4,5)$ helicity amplitudes at the same kinematic point of Table II. As only one-loop integrals are required for these amplitudes, the integration error is negligible.

\begin{tabular}{ccccc}
\hline \hline & $\hat{A}_{++++++}^{(2),[2]}$ & $\hat{A}_{-++++}^{(2),[2]}$ & $\hat{A}_{--+++}^{(2),[2]}$ & $\hat{A}_{-++++}^{(2),[2]}$ \\
\hline$\epsilon^{0}$ & 3.6255 & -0.0664 & 0.2056 & 0.0269 \\
\hline \hline
\end{tabular}

effect of the highest-rank tensor integrals which appear only in the latter case. We find a convincing agreement between the poles and our amplitudes within the numerical integration error [60]. Since the full amplitude is the sum of all three parts, we see in this case that the simple $\left(d_{s}-2\right)^{0}$ part dominates and the complete amplitude is evaluated with subpercent level accuracy. This feature is probably not generic for the whole phase-space, however.

Conclusions.-The techniques presented in this Letter have allowed the first look at a set of five-point two-loop helicity amplitudes with phenomenological relevance for LHC experiments. We have found that unitarity cutting methods in six dimensions can be combined with finite field reconstruction techniques to compute multiscale dimensionally regulated two-loop amplitudes in QCD. In many cases, it was possible to obtain completely analytic expressions for the integrands of the helicity amplitudes.

While a lot of effort was taken to find manageable expressions, the final integrand form was still extremely large and significantly more challenging than the previously known all-plus helicity configuration. One obvious next step is to include a full set of integration-by-parts identities and reduce the amplitude onto a basis of analytically computed MIs. Promising new approaches that use finite field reconstruction [61] or algebraic geometry analyses [27,62-65] could make this possible in the near future. We expect there will be other ways to improve the integrand form by using canonical bases [29] and local integrand representations [66-68], though at the present time more work is needed to investigate these approaches.

While there clearly remains a long list of tasks to be completed before predictions of $2 \rightarrow 3$ scattering at NNLO in QCD become a reality, the work presented here is the first example of the evaluation of one of the key ingredients. We hope that the techniques and benchmark results presented here will provide a platform towards this final goal.

We thank Fabrizio Caola, Johannes Henn, Claude Duhr, Donal O'Connell, Nigel Glover, Adriano Lo Presti, Francesco Buciuni, and Johannes Schlenk for many stimulating discussions. We particularly thank Claude Duhr for providing computer-readable expressions for the integrals in Ref. [51]. It is also a pleasure to thank the organizers of the AMPDEV2017 program at the Mainz Institute for Theoretical Physics (MITP), Mainz and also the organizers of the "Automated, Resummed and Effective: Precision Computations for the Large Hadron Collider (LHC) and Beyond" and "Mathematics and Physics of Scattering Amplitudes" programs at the Munich Institute for Astroand Particle Physics (MIAPP) center, Munich for creating stimulating working environments while this work was ongoing. This work is supported by STFC Fellowship No. ST/L004925/1 and Grant No. ST/M004104/1. The work of T. P. has received funding from the European Research Council (ERC) under the European Union's Horizon 2020 research and innovation program (Grant Agreement No. 725110). We are also indebted to Samuel Abreu, Fernando Febres Cordero, Harald Ita, Ben Page, and Mao Zeng for pointing out typographical errors in a previous version of this Letter.

*simon.d.badger@durham.ac.uk

[1] E. W. N. Glover, C. Oleari, and M. E. Tejeda-Yeomans, Nucl. Phys. B605, 467 (2001).

[2] Z. Bern, A. De Freitas, and L. J. Dixon, J. High Energy Phys. 03 (2002) 018.

[3] Z. Bern, L. J. Dixon, and D. A. Kosower, Phys. Rev. Lett. 70, 2677 (1993).

[4] Z. Bern, L. J. Dixon, D. C. Dunbar, and D. A. Kosower, Nucl. Phys. B425, 217 (1994).

[5] Z. Bern, L. J. Dixon, D. C. Dunbar, and D. A. Kosower, Nucl. Phys. B435, 59 (1995).

[6] R. Britto, F. Cachazo, and B. Feng, Nucl. Phys. B725, 275 (2005).

[7] Z. Bern, L. J. Dixon, and D. A. Kosower, Nucl. Phys. B513, 3 (1998).

[8] G. Ossola, C. G. Papadopoulos, and R. Pittau, Nucl. Phys. B763, 147 (2007).

[9] P. Mastrolia and G. Ossola, J. High Energy Phys. 11 (2011) 014.

[10] P. Mastrolia, E. Mirabella, G. Ossola, and T. Peraro, Phys. Lett. B 718, 173 (2012).

[11] P. Mastrolia, E. Mirabella, G. Ossola, and T. Peraro, Phys. Rev. D 87, 085026 (2013).

[12] P. Mastrolia, E. Mirabella, G. Ossola, and T. Peraro, Phys. Lett. B 727, 532 (2013).

[13] S. Badger, H. Frellesvig, and Y. Zhang, J. High Energy Phys. 04 (2012) 055.

[14] Y. Zhang, J. High Energy Phys. 09 (2012) 042.

[15] S. Badger, H. Frellesvig, and Y. Zhang, J. High Energy Phys. 12 (2013) 045.

[16] S. Badger, G. Mogull, A. Ochirov, and D. O'Connell, J. High Energy Phys. 10 (2015) 064.

[17] S. Badger, G. Mogull, and T. Peraro, J. High Energy Phys. 08 (2016) 063.

[18] T. Gehrmann, J. M. Henn, and N. A. Lo Presti, Phys. Rev. Lett. 116, 062001 (2016); 116, 189903(E) (2016).

[19] D. C. Dunbar and W. B. Perkins, Phys. Rev. D 93, 085029 (2016).

[20] D. C. Dunbar, G. R. Jehu, and W. B. Perkins, Phys. Rev. D 93, 125006 (2016). 
[21] D. C. Dunbar and W. B. Perkins, Phys. Rev. Lett. 117, 061602 (2016).

[22] D. C. Dunbar, J. H. Godwin, G. R. Jehu, and W. B. Perkins, Phys. Rev. D 96, 116013 (2017).

[23] D. A. Kosower and K. J. Larsen, Phys. Rev. D 85, 045017 (2012).

[24] S. Caron-Huot and K. J. Larsen, J. High Energy Phys. 10 (2012) 026.

[25] S. Abreu, F. F. Cordero, H. Ita, M. Jaquier, B. Page, and M. Zeng, Phys. Rev. Lett. 119, 142001 (2017).

[26] S. Abreu, F. Febres Cordero, H. Ita, M. Jaquier, and B. Page, Phys. Rev. D 95, 096011 (2017).

[27] H. Ita, Phys. Rev. D 94, 116015 (2016).

[28] C. G. Papadopoulos, D. Tommasini, and C. Wever, J. High Energy Phys. 04 (2016) 078.

[29] J. M. Henn, Phys. Rev. Lett. 110, 251601 (2013).

[30] C. G. Papadopoulos, J. High Energy Phys. 07 (2014) 088.

[31] T. Peraro, J. High Energy Phys. 12 (2016) 030.

[32] A. Hodges, J. High Energy Phys. 05 (2013) 135.

[33] Z. Bern, A. De Freitas, L. J. Dixon, and H. L. Wong, Phys. Rev. D 66, 085002 (2002).

[34] W. L. van Neerven and J. A. M. Vermaseren, Phys. Lett. 137B, 241 (1984).

[35] The renormalizability constraints restrict the maximum rank in the loop momenta that can appear in $\Delta$. This is discussed in more detail in the literature on multiloop integrand reduction.

[36] C. Cheung and D. O'Connell, J. High Energy Phys. 07 (2009) 075.

[37] F. A. Berends and W. T. Giele, Nucl. Phys. B306, 759 (1988).

[38] P. Mastrolia, T. Peraro, and A. Primo, J. High Energy Phys. 08 (2016) 164.

[39] P. S. Wang, in Proceedings of the Fourth ACM Symposium on Symbolic and Algebraic Computation (ACM, New York, 1981), pp. 212-217.

[40] P. S. Wang, M. J. T. Guy, and J. H. Davenport, SIGSAM Bulletin 16, 2 (1982).

[41] ISSAC '06: Proceedings of the 2006 International Symposium on Symbolic and Algebraic Computation (ACM, New York, 2006), p. 505060.

[42] M. Kauers, Nucl. Phys. B, Proc. Suppl. 183, 245 (2008).

[43] P. Nogueira, J. Comput. Phys. 105, 279 (1993).
[44] J. Kuipers, T. Ueda, J. A. M. Vermaseren, and J. Vollinga, Comput. Phys. Commun. 184, 1453 (2013).

[45] B. Ruijl, T. Ueda, and J. Vermaseren, arXiv:1707.06453.

[46] Z. Bern, M. Czakon, D. A. Kosower, R. Roiban, and V. A. Smirnov, Phys. Rev. Lett. 97, 181601 (2006).

[47] O. V. Tarasov, Phys. Rev. D 54, 6479 (1996).

[48] R. N. Lee, Nucl. Phys. B830, 474 (2010).

[49] A. V. Smirnov, Comput. Phys. Commun. 204, 189 (2016).

[50] S. Borowka, G. Heinrich, S. Jahn, S. P. Jones, M. Kerner, J. Schlenk, and T. Zirke, Comput. Phys. Commun. 222, 313 (2018).

[51] T. Gehrmann and E. Remiddi, Nucl. Phys. B601, 248 (2001).

[52] A. V. Smirnov, Comput. Phys. Commun. 189, 182 (2015).

[53] A. von Manteuffel and C. Studerus, arXiv:1201.4330.

[54] R. N. Lee, arXiv:1212.2685.

[55] S. Catani, Phys. Lett. B 427, 161 (1998).

[56] T. Becher and M. Neubert, J. High Energy Phys. 06 (2009) 081; 11 (2013) 024(E).

[57] T. Becher and M. Neubert, Phys. Rev. Lett. 102, 162001 (2009); 111, 199905(E) (2013).

[58] E. Gardi and L. Magnea, J. High Energy Phys. 03 (2009) 079.

[59] C. Gnendiger, A. Signer, and D. Stöckinger, Phys. Lett. B 733, 296 (2014).

[60] The uncertainty on the finite terms in Tables II and III is a rough estimate made by comparing FIESTA evaluations with different numbers of sample points.

[61] A. von Manteuffel and R. M. Schabinger, Phys. Lett. B 744, 101 (2015).

[62] J. Gluza, K. Kajda, and D. A. Kosower, Phys. Rev. D 83, 045012 (2011).

[63] K. J. Larsen and Y. Zhang, Phys. Rev. D 93, 041701 (2016).

[64] A. Georgoudis, K. J. Larsen, and Y. Zhang, Comput. Phys. Commun. 221, 203 (2017).

[65] Z. Bern, M. Enciso, H. Ita, and M. Zeng, Phys. Rev. D 96, 096017 (2017).

[66] N. Arkani-Hamed, J. L. Bourjaily, F. Cachazo, S. CaronHuot, and J. Trnka, J. High Energy Phys. 01 (2011) 041.

[67] N. Arkani-Hamed, J. L. Bourjaily, F. Cachazo, and J. Trnka, J. High Energy Phys. 06 (2012) 125.

[68] J. L. Bourjaily, E. Herrmann, and J. Trnka, J. High Energy Phys. 06 (2017) 059. 